\title{
BMJ Open Retrospective cohort study of the relationship between systolic blood pressure variability and multiple sclerosis disability
}

\author{
Myla D Goldman (D) , ${ }^{1}$ Seulgi Min, ${ }^{2}$ Jennifer M Lobo, ${ }^{3}$ Min-Woong Sohn ${ }^{4}$
}

To cite: Goldman MD, Min S, Lobo JM, et al. Retrospective cohort study of the relationship between systolic blood pressure variability and multiple sclerosis disability. BMJ Open 2020;10:e034355. doi:10.1136/ bmjopen-2019-034355

- Prepublication history and additional material for this paper are available online. To view these files, please visit the journal online (http://dx.doi. org/10.1136/bmjopen-2019034355).

Received 16 September 2019 Revised 20 January 2020 Accepted 29 January 2020
Check for updates

(C) Author(s) (or their employer(s)) 2020. Re-use permitted under CC BY-NC. No commercial re-use. See rights and permissions. Published by BMJ.

${ }^{1}$ Neurology, Virginia Commonwealth University, Richmond, Virginia, USA ${ }^{2}$ Neurology, University of Virginia, Charlottesville, Virginia, USA ${ }^{3}$ Public Health Sciences, University of Virginia, Charlottesville, Virginia, USA ${ }^{4}$ Health Management and Policy, University of Kentucky, Lexington, Kentucky, USA

Correspondence to Myla D Goldman; myla.goldman@vcuhealth.org

\section{ABSTRACT}

Objective To examine the relationship between visitto-visit systolic blood pressure (SBP) variability and patient-reported outcome measure of disability in multiple sclerosis (MS) patients.

Design A retrospective cohort study of individuals with MS who completed a patient-determined disease steps (PDDS) scale between 2011 and 2015 at an MS specialty clinic.

Participants Individuals with MS for whom both a completed PDDS scale and $\geq 3$ SBP measures within the prior 12 months of the survey were available.

Main outcome measure Participants were grouped into three classes of disability (no or mild (PDDS 0-1), moderate (2-3), severe (4-7)). SBP variability was calculated as within-subject SD using all SBP measures taken during the past 12 months. SBP variability was analysed by Tertile groups.

Results Ninety-two subjects were included in this analysis. Mean PDDS score was $2.22 \pm 1.89$. Compared with subjects in Tertile 1 (lowest variability), the odds of being in a higher disability group was 3.5 times higher (OR=3.48; 95\% Cl: 1.08 to $11.25 ; p=0.037)$ in Tertile 2 and 5.2 times higher $(\mathrm{OR}=5.19 ; 95 \% \mathrm{Cl}: 1.53$ to 17.61 ; $\mathrm{p}=0.008$ ) in Tertile 3 (highest variability), independent of mean SBP, age, sex, race/ethnicity, body mass index and comorbidities ( $p$ for trend $=0.008$ ). Mean PDDS scores were 1.52 \pm 1.18 in Tertile 1, 2.73 \pm 1.02 in Tertile 2 and $2.42 \pm 0.89$ in Tertile 3 after adjusting for the same covariates.

Conclusions Our results show a significant gradient relationship between SBP variability and MS-related disability. More research is needed to determine the underlying pathophysiological relationship between SBP variability and MS disability progression.

\section{INTRODUCTION}

Multiple sclerosis (MS) is an inflammatory and degenerative disorder of the central nervous system. Individuals with MS commonly experience some degree of disability progression independent of inflammatory-driven events. The underlying mechanisms driving this inflammatory-independent disease progression remains poorly understood. It is likely that there is no single factor that

\section{Strengths and limitations of this study}

- This is the first study to look at the relationship between the systolic blood pressure variability and multiple sclerosis-related disability outcomes.

- This study paired prospectively collected patientreported outcomes with retrospectively collected data, which allowed us to leverage existing data to take a first look at this novel question.

- Our analysis included a multifaceted approach including patient-reported measures, clinical outcomes (blood pressure) and concurrent comorbid diagnosis.

- The retrospective collection of the paired clinical data limited the standardisation of the number and interval timing of blood pressure measurements, as well as the total number of subjects available for analysis.

drives MS progression. Instead, it is believed to be a multifaceted process with variable importance and influence of factors for any individual person. Posited factors include medical comorbidities, as well as environmental factors such as smoking or vitamin D exposure.

Comorbid cardiovascular disease (CVD) is more prevalent in MS relative to healthy populations. In MS patients, CVD is associated with worsened disease progression and reduced quality of life, although the mechanism remains uncertain. ${ }^{1-5}$ Visit-to-visit systolic blood pressure (SBP) variability is an emerging risk factor for a wide array of health outcomes, including CVD, kidney failure, cognitive dysfunction, diabetic complications and all-cause mortality. ${ }^{6-10}$ Excessive SBP variability $(\geq 10$ within-subject $\mathrm{SD})$ has been associated with many of these outcomes independent of mean blood pressure and hypertension. ${ }^{8} 1112$ Evidence suggests that visit-to-visit blood pressure variability may have stronger effects on cardiovascular outcomes than that of measures taken during a single 
visit or by 24 hours ambulatory monitoring devices. ${ }^{13-15}$ While various vascular comorbidities have been previously studied in the progression of MS, the relationship between SBP variability and MS progression has yet to be explored.

We conducted a retrospective cohort study to examine the relationship between SBP variability and self-reported MS disability. We hypothesised that higher SBP variability is associated with greater degree of disability among individuals with MS.

\section{MATERIAL AND METHODS \\ Study design and sample}

We conducted a retrospective cohort study of individuals with MS who participated in research between 2011 and 2015 at the University of Virginia (UVA) School of Medicine and had previously prospectively completed the patient-determined disease steps (PDDS) scale, a validated patient-reported outcome measure of MS disability. ${ }^{16-18}$ The PDDS is a self-report tool of MS disability in which participants indicate their level of disability between 0 ('normal') and 8 ('bedridden'), where 4 indicates 'early cane' use. SBP measurements were obtained from medical records and only those subjects with $\geq 3$ available SBP measurements captured within the 12 months prior to PDDS completion were included in the analysis. This study was approved by the UVA institutional review board.

\section{Visit-to-visit variability of SBP}

All available SBP measures within 12 months pre-PDDS and post-PDDS survey data were extracted from the electronic medical records system. Within-subject means and SD of SBP were computed. Coefficient of variation was calculated by dividing the SD by the mean to obtain a measure of variability that was more independent of the mean than SD. We used the within-subject coefficients of variation (SBPCV) to divide the study sample into three equal-sized groups (tertiles), whose SBPCV ranges are $0.012-0.064$ for Tertile 1 (the lowest variability group), $0.065-0.087$ for Tertile 2 and $0.089-0.172$ for Tertile 3 (the highest variability group).

\section{Covariates}

Demographic data (age, sex and race/ethnicity) were collected. We searched with the Clinical Data Repository, a data warehouse containing clinical information from patients treated at the UVA, for the 12-month period prior to the PDDS survey to identify coexisting conditions including CVD (ICD-9-CM codes, 410.xx - 414.xx, 428.xx, 431.xx, 434.xx and 436.xx), peripheral vascular disease (443.9), diabetes (250.xx, 357.2 and 362.01), depression (311.xx, 300.4, 296.20, 296.80, 296.89 and 296.90) and hypertension (401.x). In addition to the diagnostic codes, we classified hypertension in patients using the 140/90 mm Hg per American College of Cardiology/ American Heart Association (ACC/AHA) guideline. ${ }^{19}$
We also extracted body mass index (BMI) data within 6 months of the PDDS survey completion date.

\section{Statistical analysis}

We used multivariable regression analysis to examine the relationship between SBP variability and the PDDS disability rating. To best utilise the ordinal nature of our response variable (PDDS score), ${ }^{16}{ }^{17}$ we estimated an ordinal logistic regression ${ }^{20}$ and found that it did not satisfy the proportional odds assumption. ${ }^{20-22}$ We tried several medically meaningful groupings to satisfy the assumption based on the PDDS scores and decided on three groups that make psychological and medical sense as distinctive groups as follows: no or mild disability (PDDS scores 0 or 1 ), moderate disability (PDDS scores 2 or 3 ) and severe disability (PDDS scores 4 or higher). The disability outcomes in these new groups were modelled using ordinal logistic regression as a function of SBP variability, adjusting for patient demographic data (age, sex and race/ethnicity), mean SBP, BMI, hypertension and depression.

As a sensitivity analysis, we defined the PDDS score 3 or above as the presence of moderate or severe disability and modelled the binary response $(0=$ no or mild disability; $1=$ moderate to severe disability) using a logistic regression (online supplementary table e-1). Because SBP variability is found to be correlated with the number of measures used in computing the within-subject SD, we controlled for the number of BP measures in another sensitivity analysis (online supplementary table e-2).

Finally, we tested whether PDDS scores can predict SBP variability before the study (online supplementary table e-3) and whether PDDS scores can predict SBP variability after the survey (online supplementary table e-4) by estimating linear regressions to predict presurvey and postsurvey SBP variability as a function of PDDS scores, adjusting for age, sex, race and other covariates.

We used Stata SE V.15.1 for all statistical analysis.

\section{Patient and public involvement}

No patient involved.

\section{RESULTS}

A total of 218 PDDS surveys were identified from available study data. Among these, 17 subjects had completed more than one PDDS survey; in such cases, the first available survey date with corresponding $\geq 3$ SBP measures was utilised. No subject contributed more than once to the final data set. When the same respondent participated in the PDDS survey more than once, we used the first survey. Of the resultant subjects, only 94 had the requisite $\geq 3$ blood pressure measures in the 12 months prior to the survey completion date. Two additional subjects were excluded due to lack of available records to permit BMI calculation (absent height and/or weight).

The resultant 92 subjectsincluded in the final analysis had a mean age of $44.7 \pm 12.2$ years at the time of PDDS survey 
Table 1 Characteristics of study cohort $(n=92)$

\begin{tabular}{|c|c|c|c|c|c|}
\hline \multirow[b]{3}{*}{ Variable } & \multicolumn{5}{|c|}{ Tertiles of SBP coefficient of variation } \\
\hline & \multirow{2}{*}{$\begin{array}{l}\text { All } \\
\mathrm{N}(\%)\end{array}$} & \multirow{2}{*}{$\begin{array}{l}1 \text { (lowest } \\
\text { variability) } \\
\mathrm{N}(\%)\end{array}$} & \multirow{2}{*}{$\begin{array}{l}2 \\
N(\%)\end{array}$} & \multirow{2}{*}{$\begin{array}{l}3 \text { (highest } \\
\text { variability) } \\
\mathbf{N}(\%) \\
\end{array}$} & \multirow[b]{2}{*}{$P$ value } \\
\hline & & & & & \\
\hline All, n (row \%) & $92(100.00 \%)$ & $31(33.70 \%)$ & $30(32.61 \%)$ & $31(33.70 \%)$ & \\
\hline Age, mean (SD) & $44.71(12.16)$ & $45.03(14.29)$ & $45.93(12.54)$ & $43.19(9.41)$ & 0.673 \\
\hline Female & $50(54.35 \%)$ & $18(58.06 \%)$ & $20(66.67 \%)$ & $12(38.71 \%)$ & 0.08 \\
\hline White race & $76(82.61 \%)$ & 29 (93.55\%) & 25 (83.33\%) & $22(70.97 \%)$ & 0.063 \\
\hline \multicolumn{6}{|l|}{ Within-subject SBP } \\
\hline Mean (mm Hg), mean (SD) & $124.05(13.19)$ & $128.01(12.98)$ & $118.16(11.86)$ & $125.78(13.00)$ & 0.008 \\
\hline $\mathrm{SD}(\mathrm{mm} \mathrm{Hg})$, mean $(\mathrm{SD})$ & $9.94(4.59)$ & $5.82(2.05)$ & $9.17(1.41)$ & $14.79(3.91)$ & $<0.001$ \\
\hline Maximum (mm Hg), mean (SD) & $137.95(15.11)$ & $135.74(13.70)$ & $132.60(12.37)$ & $145.32(16.34)$ & 0.002 \\
\hline Minimum (mm Hg), mean (SD) & $110.68(14.19)$ & $120.45(13.11)$ & $105.43(11.24)$ & 106.00 (12.95) & $<0.001$ \\
\hline Number of measures, mean (SD) & $7.93(5.53)$ & $6.29(3.97)$ & $10.33(6.18)$ & $7.26(5.57)$ & 0.011 \\
\hline Body mass index $\left(\mathrm{kg} / \mathrm{m}^{2}\right)$, mean $(\mathrm{SD})$ & $29.03(6.02)$ & $28.73(5.64)$ & $28.04(5.25)$ & $30.28(6.99)$ & 0.33 \\
\hline Depression & $19(20.65 \%)$ & $4(12.90 \%)$ & $11(36.67 \%)$ & $4(12.90 \%)$ & 0.031 \\
\hline Hypertension & $28(30.43 \%)$ & $13(41.94 \%)$ & $9(30.00 \%)$ & $6(19.35 \%)$ & 0.154 \\
\hline PDDS score, mean (SD) & $2.22(1.89)$ & $1.52(1.95)$ & $2.73(1.70)$ & $2.42(1.86)$ & 0.031 \\
\hline PDDS score, median (IQR) & $2(0-4)$ & $0(0-3)$ & $3(1-4)$ & $2(1-4)$ & \\
\hline \multicolumn{6}{|l|}{ PDDS score (3 groups) } \\
\hline No or mild $(0-1)$ & $40(43.48 \%)$ & 19 (61.29\%) & $9(30.00 \%)$ & $12(38.71 \%)$ & 0.163 \\
\hline Moderate (2-3) & 27 (29.35\%) & $6(19.35 \%)$ & $11(36.67 \%)$ & $10(32.26 \%)$ & \\
\hline Severe (4 or higher) & $25(27.17 \%)$ & $6(19.35 \%)$ & 10 (33.33\%) & $9(29.03 \%)$ & \\
\hline
\end{tabular}

All percentages are either column percentages (col. \%) or row percentages (row \%). P-values for continuous variables were computed using one-way analysis of variance and those for categorical variables were based on Pearson $\chi^{2}$ tests.

PDDS, patient-determined disease steps; SBP, systolic blood pressure.

completion. They were predominantly white $(82.6 \%)$ and $54 \%$ female. Their mean SBP was $124.1 \pm 13.2 \mathrm{~mm} \mathrm{Hg}$ overall and were highest in Tertile $1(128.0 \pm 13.0 \mathrm{~mm} \mathrm{Hg})$ and lowest in Tertile $2(118.2 \pm 11.9 \mathrm{~mm} \mathrm{Hg})$. Their withinsubject SBP SD was $9.9 \pm 4.6 \mathrm{~mm} \mathrm{Hg}$ overall but changed from $5.8 \pm 2.1 \mathrm{~mm} \mathrm{Hg}$ (IQR 4.4-7.4 $\mathrm{mm} \mathrm{Hg}$ ) to $9.2 \pm 1.4 \mathrm{~mm}$ $\mathrm{Hg}$ (IQR 8.5-10.2 mm Hg) in Tertile 2 and 14.8 \pm 3.9 (IQR $11.7-17.7 \mathrm{~mm} \mathrm{Hg}$ ) in Tertile 3. Their mean BMI was $29.0 \mathrm{~kg} / \mathrm{m}^{2}$. A total of $19(20.7 \%)$ had depression, 28 (30.4\%) had hypertension (11 patients with a diagnosis in ICD-9-CM and 17 patients with elevated mean BP). We could not identify any subject with vascular comorbidities except for one who had acute myocardial infarction and was in Tertile 2. For this reason, vascular comorbidities have not been used in any subsequent analyses. The mean and median PDDS score was $2.2 \pm 1.89$ and 2 (IQR $0-4)$. Forty patients $(43.5 \%)$ had no or mild disability, $27(29.4 \%)$ had moderate disability and $25(27.2 \%)$ had severe disability (table 1 ).

Participants included in the analysis were not significantly different from those excluded $(n=126)$ in terms of PDDS score, patient sex, race and BMI (table 2). However, included subjects were older ( 48.7 vs 44.7 years; $p=0.016$ ), less hypertensive (30.4\% vs $52.4 \%$; $\mathrm{p}=0.001$ ) and more depressed ( $20.7 \%$ vs $5.6 \%$; $\mathrm{p}<0.001)$.

Results from ordinal logistic regression analyses are shown in table 3. Compared with subjects in Tertile 1 (lowest variability), the odds of being in a higher disability group was 3.5 times higher $(\mathrm{OR}=3.48 ; 95 \% \mathrm{CI}$ : 1.08 to $11.25 ; \mathrm{p}=0.037$ ) in Tertile 2 and 5.2 times higher $(\mathrm{OR}=5.19 ; 95 \% \mathrm{CI}: 1.53$ to $17.62 ; \mathrm{p}=0.008)$ in Tertile 3 (highest variability), independent of mean SBP, age, sex, race/ethnicity, BMI, hypertension and depression ( $\mathrm{p}$ for trend $=0.008$ ). Mean PDDS scores were $1.52 \pm 1.18$ in Tertile 1, 2.73 \pm 1.02 in Tertile 2 and $2.42 \pm 0.89$ in Tertile 3 after adjusting for the same covariates as the model shown in table 3 .

For sensitivity analysis, we checked the robustness of this association by estimating a logistic regression that predicted the binary indicator of PDDS score 3 or above (moderate or severe disability) (online supplementary table e-1). The sensitivity analysis showed a significant relationship between SBP variability and disability ratings assessed by PPDS scale persisted.

We checked whether the number of SBP measures used to compute the variability is a confounding factor between 
Table 2 Comparison of the included and excluded patients in the original cohort

\begin{tabular}{|c|c|c|c|c|}
\hline & All & Excluded & Included & \\
\hline Variables & $\mathrm{N}$ (col. \%) & N (row \%) & N (row \%) & $P$ value \\
\hline All & $218(100.00 \%)$ & $126(57.80 \%)$ & $92(42.20 \%)$ & \\
\hline Age, mean (SD) & $47.04(12.24)$ & $44.71(12.16)$ & $48.74(12.06)$ & 0.016 \\
\hline Female & $113(51.83 \%)$ & $63(50.00 \%)$ & $50(54.35 \%)$ & 0.526 \\
\hline White race & $181(83.03 \%)$ & 105 (83.33\%) & $76(82.61 \%)$ & 0.888 \\
\hline BMI $\left(\mathrm{kg} / \mathrm{m}^{2}\right)$, mean (SD) & $28.25(6.19)$ & $29.03(6.02)$ & $27.59(6.29)$ & 0.102 \\
\hline Hypertension & $94(43.12 \%)$ & $66(52.38 \%)$ & $28(30.43 \%)$ & 0.001 \\
\hline Depression & $26(11.93 \%)$ & $7(5.56 \%)$ & $19(20.65 \%)$ & $<0.001$ \\
\hline PDDS score, mean (SD) & $2.05(1.81)$ & $2.22(1.89)$ & $1.93(1.75)$ & 0.247 \\
\hline \multicolumn{5}{|l|}{ PDDS score (3 groups) } \\
\hline No or mild $(0-1)$ & 95 (43.58\%) & 55 (43.65\%) & $40(43.48 \%)$ & 0.212 \\
\hline Moderate (2-3) & 75 (34.40\%) & 48 (38.10\%) & 27 (29.35\%) & \\
\hline Severe (4 or higher) & 48 (22.02\%) & 23 (18.25\%) & 25 (27.17\%) & \\
\hline
\end{tabular}

BMI, body mass index; PDDS, patient-determined disease steps.

the variability and the PDDS outcome by estimating the model shown in table 2 with the number of measures as an additional covariate (online supplementary table e-2). The significant gradient relationship persisted in this model as well ( $\mathrm{p}$ for trend $=0.007$ ).

Finally, we tested the potential multidirectionality of the relationship between PDDS scores and SBP variability by predicting the SBP variability before and after the study using PDDS scores. From the 92 included subjects, 89 subjects had available $\geq 3$ postsurvey SBP measures, for whom the presurvey and postsurvey SBP coefficients of variation were correlated at $r=0.10 \quad(p=0.349)$, while SBP means were correlated at $\mathrm{r}=0.83(\mathrm{p}<0.001)$. We estimated two regression models that predict presurvey and postsurvey SBP coefficient of variation using PDDS scores, after controlling for age, sex and race. PDDS scores did not predict presurvey variability in any model specification (online supplementary table e-3). On the other hand, those with moderate disability had 0.03 higher postsurvey coefficient of variation in SBP $(95 \%$ CI 0.01 to $0.05 ; \mathrm{p}=0.003$ ) compared with those with no or mild disability but the severe disability group did not have significantly different SBP variability from the no or mild group. Mean SBP, number of BP measures or any other comorbidities did not change this association (online supplementary table e-4). These tests of directionality of the association between SBP variability and PDDS scores are summarised in figure 1.

\section{DISCUSSION}

Our results demonstrate a significant and strong graded relationship between SBP variability and self-reported disability outcome measures (PDDS) among MS patients.

Table 3 Ordinal logistic regression results for MS patients in a higher disability group $(n=92)^{*}$

Variables

Estimate $(95 \% \mathrm{Cl})$

$P$ value

Tertiles of SBP coefficient of variation(1 (lowest variability))

2

3 (highest variability)

Age

Female (male)

White race (other races/ethnicity)

Within-subject mean SBP $(\mathrm{mm} \mathrm{Hg})$

Hypertension

Depression

Body mass index $\left(\mathrm{kg} / \mathrm{m}^{2}\right)$

\begin{tabular}{lr}
$3.48(1.077$ to 11.251$)$ & 0.037 \\
\hline $5.193(1.531$ to 17.616$)$ & 0.008 \\
\hline $1.1(1.051$ to 1.150$)$ & $<0.001$ \\
$3.177(1.249$ to 8.078$)$ & 0.015 \\
$1.495(0.450$ to 4.963$)$ & 0.512 \\
0.991 (0.952 to 1.031$)$ & 0.647 \\
0.93 (0.356 to 2.430$)$ & 0.882 \\
$1.183(0.426$ to 3.289$)$ & 0.747 \\
$1.057(0.974$ to 1.147$)$ & 0.186
\end{tabular}

${ }^{*}$ Reference categories are in angle brackets. Disability groups were defined as no or mild (PDDS scores 0 or 1$)$, moderate (2 or 3 ) and severe (4 or higher).

MS, multiple sclerosis ; SBP, systolic blood pressure. 


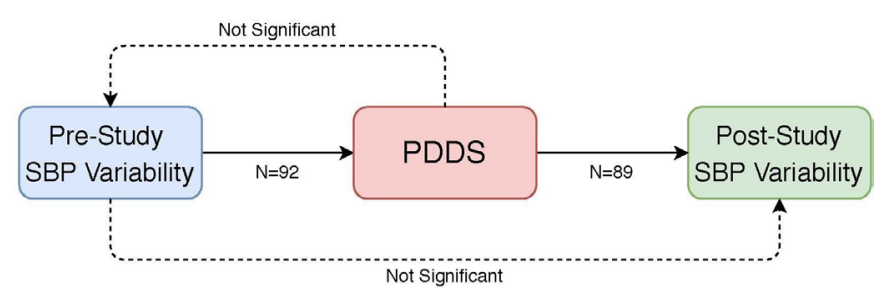

Figure 1 Summary of the significant relationships (solid arrows) and non-significant relationships (dashed arrows) between SBP variability and PDDS scores*. *Presurvey SBP variability was significantly predictive of PDDS scores $(p=0.015)$, and PDDS scores were predictive of postsurvey PDDS variability $(p=0.011)$. PDDS scores did not predict presurvey SBP variability, and presurvey SBP variability did not predict postsurvey SBP variability. The $p$-values were obtained from a Wald test with 2 degrees of freedom (presurvey variability to PDDS) and from an F test with 2 and 83 degrees of freedom (PDDS to postsurvey variability). PDDS, patient determined disease steps; SBP, systolic blood pressure.

Patients in Tertile 3 (highest variability) had an approximately six times higher risk of being in the higher disability group compared with those in Tertile 1 (lowest variability). This relationship was independent of mean SBP, BMI, hypertension, depression and patient demographic factors. This result was robust to different analytic methods such as logistic regression to predict PDDS score 3 or higher (presence of moderate to severe disability).

Another important finding in this study is that the association of excessive SBP variability with higher PDDS scores can occur in normotensive individuals. Indeed, overall, $70 \%$ of our cohort were normotensive $(<140 / 90 \mathrm{~mm} \mathrm{Hg})$ or without hypertension diagnosis. They also had lower rates of hypertension in higher SBP variability tertiles with the lowest proportion observed in Tertile 3 (19\% vs $41 \%$ in Tertile 1), a group with the highest SBP variability. This finding is consistent with previous studies by Sohn and his colleagues on diabetic complications. ${ }^{81112}$ Our results also demonstrate that mean SBP was not significantly associated with PDDS groups, suggesting that there may be a different physiologic mechanism at play, not simply elevated blood pressures. ${ }^{13}$

Excessive visit-to-visit SBP variability has been associated with cardiovascular and several other health outcomes. To our knowledge, this is the first study to show that excessive visit-to-visit SBP variability may be a risk factor for MS disability progression. Previously, several large studies have identified a relationship between vascular comorbidities and MS outcomes, both clinical and patientreported, using diagnostic codes (eg, hypertension) or medications (antihypertensives) to classify patients. ${ }^{1-4}$ Our results confirm the previous diagnosis-based research and extends that work, by identifying excessive SBP variability as a contributing factor to the previously identified relationship between blood-pressure changes and MS. Our results further suggest that a relevant haemodynamic mechanism in the interplay between CVD and
MS disability progression, is not simply hypertension (ie, elevated mean BP), but also excessive SBP variability.

Pathophysiological mechanisms involved in the relationship between blood pressure variability and health outcomes are currently explained by arterial stiffness, endothelial dysfunction and subclinical inflammation. ${ }^{23-26}$ Several factors known to increase blood pressure variability include autonomic dysfunction, ${ }^{27}$ low hydration status, ${ }^{28}$ insulin dysregulation ${ }^{29} 30$ and sleep-apena ${ }^{31}$ are commonly found in patients with MS. Tettey et al suggest that vascular comorbidities may activate the inflammatory cascade that ultimately leads to neurodegeneration which manifests in disability progression in MS. ${ }^{2}$ They also suggested that cerebral endothelial dysfunction may be involved in 'trans-endothelial migration of T-lymphocytes and monocytes to the CNS with destructive and often neurodegenerative consequences'. ${ }^{2}$ Our results suggest that excessive SBP variability could be a relevant factor in that postulated inflammatory cascade in the vasculature and that may contribute to the cerebral endothelial dysfunction, which combine to produce the MS disability progression we observed in our study. More research is needed to test whether excessive SBP variability is indeed implicated in these pathways.

It is still premature to derive any MS-related clinical implications from our results. But it is advisable that MS patients be checked for SBP variability and those with excessive variability (eg, within-subject SD of 8 or higher) be recommended for careful vascular evaluation. Interestingly, we found that the majority of patients we identified as having hypertension according to the $\mathrm{JNC}^{32}$ and 2017 ACC/AHA criteria ${ }^{19}$ did not have an actual diagnosis of hypertension. This suggests a potential underdiagnosis of hypertension, at least in our cohort.

This cross-sectional study was not designed to make any causal inferences between SBP variability and PDDS scores. However, our sensitivity analyses suggest that, while SBP variability was a strong and significant predictor of PDDS scores, the latter did not predict the former. Our data further suggest that the PDDS scores could significantly predict postsurvey SBP variability but that the presurvey and postsurvey SBP variabilities were not correlated $(\mathrm{r}=0.10 ; \mathrm{p}=0.349)$. This lends credence to the notion that SBP variability can in fact be a prognostic factor for future disability progression and that there may be a vicious cycle of increasing SBP variability and worsening disability feeding each other dynamically over time.

There are limitations to our work. This is a retrospective study in design and we relied on the Clinical Data Repository for our health system as a source of blood pressure measures and comorbid conditions. Accuracy of these values is not known. Second, we were limited in sample size, mainly because the majority of patients in the original study sample were excluded because they lacked the requisite number of SBP measures. Therefore, our results should be cautiously interpreted because of the potential for selection bias arising from requiring three or more SBP measures within 12 months prior PDDS 
measurement. However, a bivariate comparison of the included versus excluded patients in table 2 showed that they are similar in demographic factors, with the noted exception of age and depression, both of which were higher in the included population. These factors may have resulted in higher visit frequency leading to more available SBP values in those meeting eligibility criteria. Interestingly, the included population had lower incidence of hypertension compared with excluded subjects, as identified by ICD-9-CM codes or BP measures taken during the 1-year period prior to the survey completion. We were only able to capture BP measures documented in our institutional electronic medical records and there may have been additional values measured by other providers that were not captured in our data. We were not able to control for some potential confounders, including MS disease duration, disease modifying treatments and some comorbid conditions that might have affected disability outcomes in our data. In addition, while validated, PDDS is a patient-reported outcome that may have unknown response bias. Despite these limitations, we believe our results represent an important first step in studying this relationship.

In conclusion, our results show that excessive SBP variability is associated with increased disability in MS patients, independent of mean SBP, hypertension diagnosis, depression and obesity. This may represent a novel mechanism which may mediate the relationship between vascular dysfunction and progression of MS disability. Further prospective studies are needed to confirm whether excessive SBP variability is linked to the subclinical inflammation markers and/or cerebral endothelial dysfunction, and other markers of disease progression.

Contributors MDG: study concept and design, acquisition of data, analysis and interpretation of data and drafting/revising the manuscript. SM: acquisition of data, drafting/revising the manuscript. JML: diagram creation, drafting/revising the manuscript. M-WS: study concept and design, analysis and interpretation of data, statistical analysis and drafting/revising the manuscript.

Funding This work was supported by philanthropic funds provided by the ziMS Foundation.

Competing interests MDG has served as a consultant for ADAMAS, Celgene, EMD Serono, Novartis Pharmaceuticals, Sanofi Genzyme, Teva Pharmaceuticals. She has received research funding from Biogen Idec, Novartis Pharmaceuticals, National MS Society, MedDay Pharmaceuticals and PCORI.

Patient consent for publication Not required.

Provenance and peer review Not commissioned; externally peer reviewed.

Data availability statement Data are available upon reasonable request. Anonymized data not published within this article are available from the corresponding author (MDG) on reasonable request.

Open access This is an open access article distributed in accordance with the Creative Commons Attribution Non Commercial (CC BY-NC 4.0) license, which permits others to distribute, remix, adapt, build upon this work non-commercially, and license their derivative works on different terms, provided the original work is properly cited, appropriate credit is given, any changes made indicated, and the use is non-commercial. See: http://creativecommons.org/licenses/by-nc/4.0/.

ORCID iD

Myla D Goldman http://orcid.org/0000-0002-5675-945X
REFERENCES

1 Dagan A, Gringouz I, Kliers I, et al. Disability progression in multiple sclerosis is affected by the emergence of comorbid arterial hypertension. J Clin Neurol 2016;12:345-50.

2 Tettey P, Simpson S, Taylor BV, et al. Vascular comorbidities in the onset and progression of multiple sclerosis. J Neurol Sci 2014;347:23-33.

3 Marrie RA, Rudick R, Horwitz R, et al. Vascular comorbidity is associated with more rapid disability progression in multiple sclerosis. Neurology 2010;74:1041-7.

4 Conway DS, Thompson NR, Cohen JA. Influence of hypertension, diabetes, hyperlipidemia, and obstructive lung disease on multiple sclerosis disease course. Mult Scler 2017;23:277-85.

5 Marrie RA, Horwitz R, Cutter G, et al. Cumulative impact of comorbidity on quality of life in MS. Acta Neurol Scand 2012;125:180-6.

6 Hata J, Arima H, Rothwell PM, et al. Effects of visit-to-visit variability in systolic blood pressure on macrovascular and microvascular complications in patients with type 2 diabetes mellitus: the ADVANCE trial. Circulation 2013;128:1325-34.

7 Rothwell PM, Howard SC, Dolan E, et al. Prognostic significance of visit-to-visit variability, maximum systolic blood pressure, and episodic hypertension. Lancet 2010;375:895-905.

8 Sohn M-W, Epstein N, Huang ES, et al. Visit-to-visit systolic blood pressure variability and microvascular complications among patients with diabetes. J Diabetes Complications 2017;31:195-201.

9 Epstein NU, Lane KA, Farlow MR, et al. Cognitive dysfunction and greater visit-to-visit systolic blood pressure variability. J Am Geriatr Soc 2013;61:2168-73.

10 Okada H, Fukui M, Tanaka M, et al. Visit-to-visit blood pressure variability is a novel risk factor for the development and progression of diabetic nephropathy in patients with type 2 diabetes. Diabetes Care 2013;36:1908-12.

11 Budiman-Mak E, Epstein N, Brennan M, et al. Systolic blood pressure variability and lower extremity amputation in a non-elderly population with diabetes. Diabetes Res Clin Pract 2016;114:75-82.

12 Brennan MB, Guihan M, Budiman-Mak E, et al. Increasing SBP variability is associated with an increased risk of developing incident diabetic foot ulcers. J Hypertens 2018;36:2177-84.

13 Rothwell PM. Limitations of the usual blood-pressure hypothesis and importance of variability, instability, and episodic hypertension. Lancet 2010;375:938-48.

14 Mancia G, Facchetti R, Parati G, et al. Visit-to-visit blood pressure variability, carotid atherosclerosis, and cardiovascular events in the European Lacidipine Study on Atherosclerosis. Circulation 2012;126:569-78.

15 Tao Y, Xu J, Song B, et al. Short-Term blood pressure variability and long-term blood pressure variability: which one is a reliable predictor for recurrent stroke. J Hum Hypertens 2017;31:568-73.

16 Hohol MJ, Orav EJ, Weiner HL. Disease steps in multiple sclerosis: a simple approach to evaluate disease progression. Neurology 1995;45:251-5.

17 Hohol MJ, Orav EJ, Weiner HL. Disease steps in multiple sclerosis: a longitudinal study comparing disease steps and EDSS to evaluate disease progression. Mult Scler 1999;5:349-54.

18 Marrie RA, Goldman M. Validity of performance scales for disability assessment in multiple sclerosis. Mult Scler 2007;13:1176-82.

19 Whelton PK, Carey RM, The CRM. The 2017 American College of Cardiology/American heart association clinical practice guideline for high blood pressure in adults. JAMA Cardiol 2018;3:352-3.

20 Agresti A. Categorical data analysis. 3rd edn. Hoboken, NJ: Wiley, 2012.

21 Brant R. Assessing proportionality in the proportional odds model for ordinal logistic regression. Biometrics 1990;46:1171-8.

22 Agresti A. An introduction to categorical data analysis. New York: Wiley, 1996.

23 Muntner P, Whittle J, Lynch Al, et al. Visit-to-Visit variability of blood pressure and coronary heart disease, stroke, heart failure, and mortality: a cohort study. Ann Intern Med 2015;163:329-38.

24 Shimbo D, Shea S, McClelland RL, et al. Associations of aortic distensibility and arterial elasticity with long-term visit-to-visit blood pressure variability: the multi-ethnic study of atherosclerosis (MESA). Am J Hypertens 2013;26:896-902.

25 Nagai M, Hoshide S, Ishikawa J, et al. Visit-to-visit blood pressure variations: new independent determinants for carotid artery measures in the elderly at high risk of cardiovascular disease. J Am Soc Hypertens 2011;5:184-92.

26 Diaz KM, Veerabhadrappa P, Kashem MA, et al. Relationship of visitto-visit and ambulatory blood pressure variability to vascular function in African Americans. Hypertens Res 2012;35:55-61. 
27 Adamec I, Habek M. Autonomic dysfunction in multiple sclerosis. Clin Neurol Neurosurg 2013;115:S73-8.

28 Cincotta MC, Engelhard MM, Stankey M, et al. Fatigue and fluid hydration status in multiple sclerosis: a hypothesis. Mult Scler 2016;22:1438-43.

29 Penesova A, Vlcek M, Imrich R, et al. Hyperinsulinemia in newly diagnosed patients with multiple sclerosis. Metab Brain Dis 2015;30:895-901.
30 Goldman M, Koenig S, Yeamans R, et al. A study of Insuling resistance in multiple sclerosis subjects and healthy controls. American Academy of Neurology 2014;82.

31 Brass SD, Duquette P, Proulx-Therrien J, et al. Sleep disorders in patients with multiple sclerosis. Sleep Med Rev 2010;14:121-9.

32 Chobanian AV, Bakris GL, Black HR, et al. Seventh report of the joint National Committee on prevention, detection, evaluation, and treatment of high blood pressure. Hypertension 2003;42:1206-52. 